\title{
Wave propagation, breaking, and overtopping on a 2D reef: a comparative evaluation of numerical codes for tsunami modelling
}

\author{
M. Kazolea*, A. Filippini, M. Ricchiuto \\ Team CARDAMOM, Inria Bordeaux Sud-Ouest, \\ 200 av. de la vieille Tour 33405 Talence-cedex, France \\ S. Abadie, M. Martin Medina, D. Morichon \\ Université de Pau et des Pays de l'Adour, Anglet, France \\ C. Journeau, R. Marcer, K. Pons \\ Principia, La Ciotat, France \\ S. LeRoy R. Pedreros, M. Rousseau \\ BRGM, Orléans, France
}

\begin{abstract}
In the framework of the French research project TANDEM dedicated to tsunami modelling, a series of benchmarks has been set up, addressing the various stages of a tsunami event: generation, propagation, run-up and inundation. We present here the results of five codes, involving both depth-averaged Boussinesq and fully 3D Navier-Stokes equations, aimed at being applicable to tsunami modelling. The codes are evaluated on a flow involving propagation, run-up, overtopping and reflection of the waves on two-dimensional reefs, and compared with the experimental data produced from a set of laboratory experiments carried out at the O.H. Hinsdale Wave Research Laboratory, Oregon State University (OSU, see Roeber et al., 2010 and Roeber and Chung, 2012).
\end{abstract}

\section{Introduction}

Fringing reefs exist in many regions around the world. The set-up of the environment such as the extended lagoons and the steep flanks produce unique surf-zone processes such as wave breaking and abruptly transitions of the flow (from dispersion dominated to flux dominated), that are challenging to numerical modeling. Roeber et al. (2010) described two series of flume experiments at Oregon State University in 2007 and 2009 that include 198 tests with 10 twodimensional reef configurations and ranges of solitary wave height and water depth. These 198 test cases provide a database of hydraulic processes over typical reef configurations in tropical and subtropical environments. The data allows parameterization of the process to understand the effect of the reefs on surf-zone dynamics and to provide guidelines for flood hazard assessment and coastal infrastructure design. This test case has been widely used, from the numerical modeling community, the last few years (Roeber et al. 2010-2012, Tonelli et al. 2012, Kazolea et al. 2013-2014, Filippini et al. 2016) for the validation of wave models and for the understanding of the wave processes in a complex dynamic system. These experiments involve the propagation, run-up, overtopping and reflection of high amplitude solitary waves on two-dimensional reefs. Their purpose is on one hand to investigate processes related to breaking, bore formation, dispersion and passing from sub- to super-critical flows, while 
providing, on the other hand, data for validation of near-shore wave models in fringing reef. These characteristics motivated the choice of this case among the set of test-cases built within the French research project PIA-ANR TANDEM on tsunami risk and modeling along the French coasts (TANDEM=Tsunamis in the Atlantic and English chaNnel: Definition of the Effects through numerical modeling, see http://www-tandem.cea.fr). An extensive description of the case studies can be found in Roeber et al. (2010, 2012).

In this work five numerical codes are tested and compared. Three codes are based on depthaveraged Boussinesq models, SLOWS (Filippini et al. 2016, Ricchiuto 2015, Ricchiuto and Fillipini 2014) developed by Inria Bordeaux Sud-Ouest, TUCWave (Kazolea et al. 20122014, Kazolea and Delis 2013) co-developed by the Technical University of Crete and Inria Bordeaux Sud-Ouest and FUNWAVE-TVD (Shi et al., 2012) used by BRGM ; and two codes solve Navier-Stokes equations, Thetis (Abadie et al., 1998) used by the Universite de Pau et des Pays de l'Adour (UPPA) and EOLE developed by PRINCIPIA. The paper is organized as follows. The test case is presented in section 2 while the numerical models are briefly presented in section 3. Numerical results are demonstrated in section 4 and the main outcomes of the comparison are summarized in the conclusion.

\section{Presentation of the benchmark}

This study utilizes one of the most challenging test cases that examines the models ability in handling nonlinear dispersive waves together with wave breaking and bore propagation. The test is discussed in (Roeber et al., 2010) and (Roeber and Cheung, 2012). The Large Wave Flume in the O.H. Hinsdale Wave Research Laboratory, used for the experiment, has length of $104 \mathrm{~m}$ a width of $3.66 \mathrm{~m}$ and a height of $4.57 \mathrm{~m}$ with a reef crest (see figure 1). The tank includes a hydraulic piston type wave maker for the solitary wave generation. The set-up of the test includes a fore reef slope of $1 / 12$, a $0.2 \mathrm{~m}$ reef crest and a water depth of $2.5 \mathrm{~m}$. This set-up exposes the reef crest by $6 \mathrm{~m}$ and submerges the flat with $0.14 \mathrm{~m}$ of water. Several identical capacitance and acoustic wave gauges placed along the flume in order to measure the free surface elevation. The location of the 14 wave gauges (WG1-14) is depicted on figure 1 also presenting a sketch of the whole domain. It must be noted that the gauges are quite spaced out. So, while providing an interesting validation setting for the large scale behaviour of the flow, this set of data may not capture fine scale physics, especially in presence of complex wave breaking patterns with multiple splash ups and important air entrainment.

As shown on figure 1, the computational domain is $83.8 \mathrm{~m}$ long, with a rigid (reflecting) wall at the right end. The reef starts at $\mathrm{x}=25.9 \mathrm{~m}$ with a nominal slope of $1 / 12$. The actual slope is such that the height of $2.36 \mathrm{~m}$ is reached after $28.25 \mathrm{~m}$. At this station a $0.2 \mathrm{~m}$ height crest is mounted. The offshore slope of the crest is the same of the reef and the length of its plateau is of $1.25 \mathrm{~m}$. The onshore side has a slope of $1 / 15$ giving a nominal length for the crest basis of $6.65 \mathrm{~m}$. Using the actual offshore slope a crest basin of $6.64407 \mathrm{~m}$ is obtained. For the computation, the use of the nominal slope value is prescribed. This gives an offshore length of the crest slope (starting at $28.25 \mathrm{~m}$ ) of $2.4 \mathrm{~m}$. For boundary conditions, reflective wall at both ends of the domain $(x=0 \mathrm{~m}$ and $\mathrm{x}=83.7 \mathrm{~m})$ are used. The $0.75 \mathrm{~m}$ input solitary wave gives a dimensionless wave height of $\mathrm{A} / \mathrm{h}=0.3$ since the initial depth at still water is $2.5 \mathrm{~m}$. To 
simplify the boundary conditions, the solitary is placed initially at $\mathrm{x}=17.6 \mathrm{~m}$ which is in reality where the experimental data places the peak at the dimensionless time $(t \sqrt{g / d}=47.11)$. The numerical wave gauges are placed at the same position as the experimental ones. We examine here the free surface elevation recorded in WG2-WG14. The prescribed value of the mesh size used in the $\mathrm{PhD}$ of $\mathrm{V}$. Roeber (2010) is 0.05 unless otherwise specified. In the same reference a Courant number of 0.4 is prescribed. The gravity acceleration used is $9.80665 \mathrm{~m} / \mathrm{s}^{2}$ and for the friction model a value of the Manning coefficient of $0.014 \mathrm{~m} . \mathrm{s}^{-1 / 3}$ is prescribed.

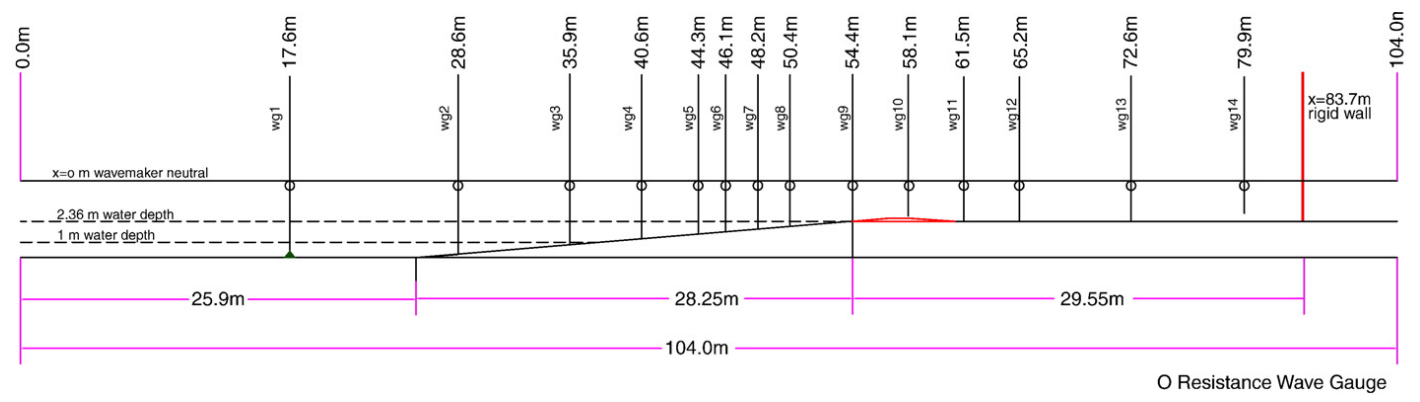

Figure 1 - Schematic of $104 \mathrm{~m}$-flume experiment over a fringing reef.

\section{Numerical models}

In this section we outline the main features of the five numerical models used in this comparison. Additional information on each of these codes can be found in the given references.

\subsection{Boussinesq-type code : TUCWave (Inria)}

TUCWave code is a high-order well-balanced unstructured finite volume (FV) scheme on triangular meshes for modelling weakly nonlinear and weakly dispersive water waves over slowly varying bathymetries (Kazolea et al. 2012 and Kazolea et al. 2014). It consists of two parts, the 1D solver and the 2D solver. The results here are obtained using the 1D part (Kazolea and Delis, 2013), in which the weakly non-linear weakly dispersive Boussinesq equations of Nwogu (1993) are solved. A formally fourth-order well-balanced hybrid finite volume/difference (FV/FD) numerical scheme for approximating the conservative form the system is used. The FV scheme is of the Godunov type and utilizes Roe's approximate Riemann solver for the advective fluxes along with well-balanced topography source term upwinding, while FD discretizations are applied to the dispersive terms in the systems. All simulations performed with TUCWave were run using the standard fourth order AdamsBashfoth/Adams-Mouton predictor corrector time integration scheme. A wave breaking mechanism of a hybrid type is also incorporated to the model. Certain criteria along with their proper implementation are established to characterize breaking waves (Kazolea et al. 2014). More precisely, we use the combination of two phase-resolving criteria for the triggering wave breaking modeling: 1 . The surface variation criterion: $\left\|\eta_{t}\right\| \geq \gamma \sqrt{g h}$ with $\gamma$ depending on the physical configuration and 2. The local slope angle criterion: $\|\nabla \eta\|_{2} \geq \tan \left(\varphi_{c}\right)$, where $\varphi_{c}$ is the critical front face angle at the initiation of the breaking. $\eta$ denotes the free surface elevation and $\mathrm{h}$ the total water depth. Once breaking waves are recognized, we switch locally in the computational domain from the BT to Nonlinear Sallow Water equations by suppressing the dispersive terms in the vicinity of the wave fronts. 


\subsection{Green-Naghdi-type code: SLOWS (Inria)}

The results of the code SLOWS (Filippini et al. 2016, Ricchiuto 2015, Ricchiuto and Filippini 2014) are obtained by solving the Green-Naghdi (G-N) equations written in the form

$\partial_{t} \eta+\partial_{t} q=0$

$\partial_{t} q+\partial_{x}(u q)+g h \partial_{x} \eta=\Phi$

$\Phi-\alpha T(\Phi)=R(\eta, u, h)$

where $q=h u$ is the integrated horizontal discharge, $h$ denotes the depth and $\eta$ is the free surface level. The source term $\Phi$ is obtained from the inversion of the elliptic operator in the last line of (1), and account for weakly dispersive-fully nonlinear effects. The model is solved by the technique proposed in Filippini et al. (2016), in which a continuous Galerkin finite element method is used for the elliptic operator, and an upwind stabilized, shock capturing scheme is used for the first two equations. A hybrid approach similar to the one implemented in TUCWave is used to model wave breaking. In this case, this boils down to locally reverting to the nonlinear shallow water equations (first two in the above system) to recover energy dissipation in breaking regions. To this scope we neglect the non-hydrostatic contribution in the hyperbolic phase imposing a tighter coupling of the two phases, with the wave breaking indicator (Kazolea et al. 2014), described also in section 3.1, embedded in the elliptic phase to smoothly turn off the dispersive effects. Time integration has been performed with the standard fourth order Adams-Bashforth/Adams Mouton predictor corrector scheme.

\subsection{Thetis (UPPA)}

The THETIS code solves the Navier-Stokes equations (NS), with assumed continuity of the velocity through the water-air interface and neglecting surface tension effects. The resulting equations read:

$\nabla \cdot u=0$

$\rho\left(\frac{\partial u}{\partial t}+(u \cdot \nabla) u\right)=\rho g-\nabla p+\nabla \cdot\left[\mu\left(\nabla u+\nabla^{\perp} u\right)\right]$

$\frac{\partial \chi}{\partial t}+u \cdot \nabla \chi=0$

In which $\chi(x, y, t)$ is a phase characteristic function equal to 1 in water and 0 in air, and $\rho$ and $\mu$ are the density and the viscosity of the fluid, respectively. They are spatially varying variables calculated using a linear interpolation based on the average value of in mixed cells. This average value is called the volume fraction F. Surface tension was not taken into account. Nevertheless the Brackbill volume formulation for surface tension (Brackbill et al. 1992) is available in THETIS, but it was not activated because the focus was not on fine scale interface deformation and dynamic but rather on wave propagation features which are not directly dependent on surface tension. The real air and water densities and viscosities are used. The equations are discretized on a fixed staggered Cartesian grid using a finite volume formulation (Patankar 1980). The coupling between velocity and pressure is solved using the augmented Lagrangian method of Fortin and Glowinski (1982). This is a minimization method under the constraint of the continuity equation, where the pressure, which is decoupled from the velocity, appears as a Lagrange multiplier. The interface displacement is solved using a Volume of Fluid (VOF) technique, which calculates the evolution of the volume fraction, occupied by one of the fluids (i.e. water) in a cell. Different VOF methods 
are implemented in the model THETIS: VOF-TVD and SVOF-PLIC. Simulations have been carried out comparing both methods for this benchmark and the closest results to the experimental data were found with the TVD scheme. The latter is only presented hereafter. VOF-TVD consists in solving equation (2) directly using a suitable numerical scheme. Using this method, and a first order discretization in time, the convective term of equation (2) is written in the conservative form. A TVD scheme typically has two main properties: in the parts of the domain where the solutions are regular, it is equivalent to a high-order scheme that reduces diffusion, and in the parts where there are strong discontinuities it is a first order scheme that prevents oscillations.

Turbulence is modeled using Large Eddy Simulation. Therefore, the viscosity which appears in equations (2) is in fact the sum of the molecular fluid viscosity and a subgrid scale viscosity, which comes from the LES filtering operation. In this paper, we used the mixed scale subgrid model detailed in Lubin et al. (2006). In this model, the subgrid viscosity is calculated as function of the resolved deformation rate tensor as in Smagorinsky's model and the fluctuating kinetic energy of the subgrid scale evaluated from the resolved field. The advantages of this subgrid model are: a subgrid viscosity depending on large scale but also on small scale flow (i.e., not like Smagorinski's model) and a model which naturally vanishes towards the wall (i.e., no need for specific treatment close to walls). The tests performed on the benchmark presented in this paper show that the turbulence modeling significantly improves the results accuracy in this particular case. More precisely the viscosity in the Navier-Stokes equations is the sum of the molecular viscosity and an additional viscosity calculated by the Large Eddy Simulation (LES) model reported by Sagaut et al. (1996). This physical additional viscosity was found to help to stabilize computations by smoothing very fine interface deformations which may appear during the simulation and which are very difficult to solve properly. The code in this work uses an irregular mesh of 323520 elements with a minimal size of $0.025 \mathrm{~m}$

The model is developed at I2M University of Bordeaux. Nevertheless the UPPA project leader contributed to the code at the beginning of its elaboration (by programming the VOF code for instance) and the UPPA team also programs their own additional routines when required.This model was used and validated in several works involving water waves (e.g., Abadie et al., 1998, Lubin et al., 2006, Abadie et al., 2010, Mory et al., 2011, Desombre et al., 2013). 


\subsection{EOLE (Principia)}

The EOLE code developed by Principia since 1990 is a multi-phase URANS model solving the equations on structured curvilinear multi-blocks meshes (possibly moving and deforming). It is based on a pseudo-compressibility technique using a dual time stepping and a second order finite volume scheme for spatial discretization (Guignard et al., 2001). The motion of the interface between the different phases is simulated from an implicit VOF model avoiding any CFL constraint and thus allowing globally large time steps. The transport of the VOF function (actually the displacement of the interface) may be ensured by a classical Eulerian equation or by an improved Eulerian-Lagrangian method developed by Principia, especially for complex wave breaking problem (Guignard et al., 2001; Biausser et al., 2004, R. Marcer et al., 2016). The surface tension is not taken into account because inertia is more important than surface tension (larger Weber number) and real air and water densities and viscosities are used in the simulations (two phase-flow simulations). The code is fully parallelized and uses MPI/OMP libraries and in this work it uses a mesh size of 333126 cells with mesh grid size $\Delta \mathrm{x}=0.05 \mathrm{~m}$ and $\Delta \mathrm{y}=0.04 \mathrm{~m}$.

We must mention that concerning all the dispersive models require high-order derivatives of the function that describes the bathymetry. If bottom topography with discontinuous derivatives is included, and the mesh is refined ( $\Delta \mathrm{x}$ goes to 0$)$, then a smoothness of the topography is needed since the second derivative of the bathymetry is not valid. But for our case and since we have a finite number of nodes the numerical diffusion introduced by the scheme helps to overcome the above problem and the results obtained are physically correct.

\subsection{FUNWAVE-TVD 2D (BRGM)}

FUNWAVE-TVD is the most recent implementation of the Boussinesq model FUNWAVE (Wei et al., 1995), initially developed and validated for coastal wave dynamics problems, but however used to perform many successful tsunami case studies. The FUNWAVE-TVD code, that solves the Boussinesq equations of Wei et al. (1995), can work both in Cartesian (Shi et al., 2012) or spherical coordinates with Coriolis effects (Kirby et al., 2012). It uses a Total Variation Diminishing (TVD) shock-capturing algorithm with a hybrid finite-volume and finite-difference scheme to more accurately simulate wave breaking and inundation by turning off dispersive terms (hence solving NLSW during breaking) once wave breaking is detected (detection based on the Froude number of the flow). The code is fully parallelized using the Message Passing Interface (MPI) protocol, using efficient algorithms allowing a substantial acceleration of the computations with the number of cores. For operational uses, FUNWAVE-TVD has received many convenient implementations, such as the use of nested grids to refine the simulations in the interest areas, or the use of Manning coefficients to characterize bottom friction.

In the frame of the U.S. National Tsunami Hazard Mitigation Program (NTHMP), FUNWAVE-TVD has been validated for both tsunami propagation and coastal impact, through an important set of analytical, laboratory and field benchmarks (Tehranirad et al., 2011). Other recent applications have allowed the validation of the model on real cases, such as the Tohoku-Oki tsunami (Grilli et al., 2013).

For completeness, Table 1 presents a summary of the differences and similarities of the codes used in this work. 


\begin{tabular}{lllll}
\hline Name & Model & $\begin{array}{l}\text { Numerical } \\
\text { scheme }\end{array}$ & $\begin{array}{l}\text { Breaking } \\
\text { closure }\end{array}$ & Parallel \\
\hline TUCWave & $\begin{array}{l}\text { Weakly } \\
\text { nonlinear } \\
\text { /weakly } \\
\text { dispersive }\end{array}$ & FV/FD & Hybrid & No \\
\hline SLOWS & $\begin{array}{l}\text { Fully } \\
\text { nonlinear/weakly } \\
\text { dispersive }\end{array}$ & FV/FE & Hybrid & No \\
& $\begin{array}{l}\text { Fully } \\
\text { nonlinear/weakly } \\
\text { dispersive }\end{array}$ & FV/FD & Hybrid & Yes \\
TVD & Navier-Stokes & FV & No & No \\
Thetis & Navier-Stokes & FV/VOF & No & Yes \\
EOLE & & & & \\
\hline
\end{tabular}

Table 1 - Summary of the CODES used in this work

\section{Large scale flow: comparison of the different models}

This section discusses the large scale features of the flow, as predicted by the different models, comparing with the experimental data. Note that for the Navier-Stokes codes (Thetis and EOLE), this entails a post-processing of the data which is quite delicate, and which will be discussed in some more detail in the next section.

We start by comparing the water level distributions along the flume at different dimensionless times $\mathrm{t}^{*}=\mathrm{t} \sqrt{\mathrm{g} / d}$. For sake of clarity, we have selected fewer snapshots than those discussed in (Roeber, 2010) and (Roeber and Cheung, 2012), representative of the propagation, breaking, and overtopping phases. The numerical results are compared to the experimental data on figures 2(a) to 2(i). For easier reading, the results have been split in two sets one involving all the Boussinesq models, and the other the two Navier-Stokes codes.
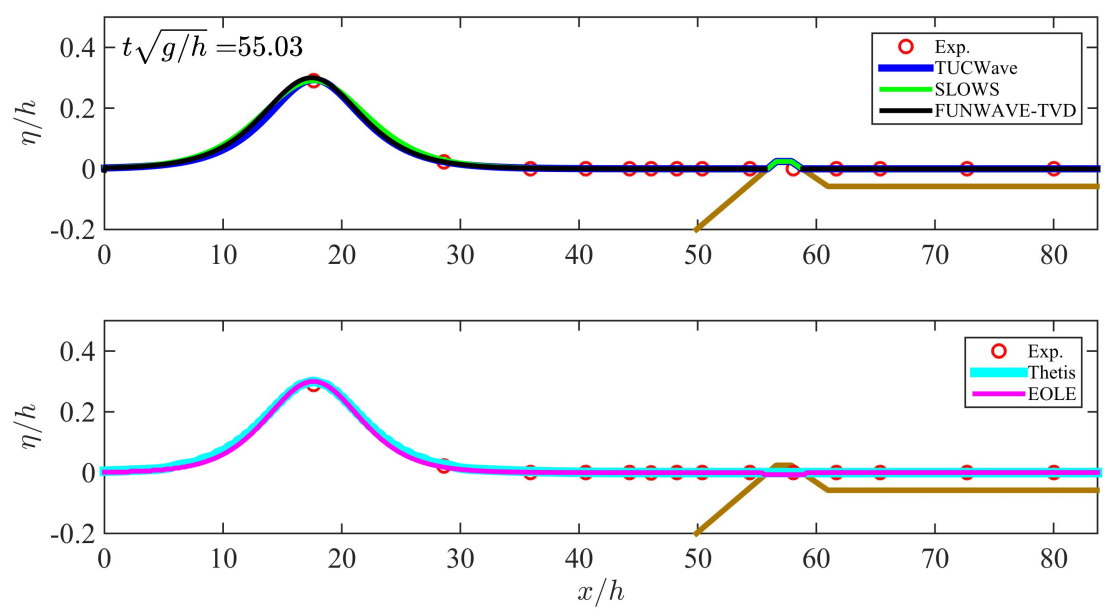

Figure 2(a) - Large scale flow behaviour. Water level at $\mathrm{t}^{*}=55.03$ 

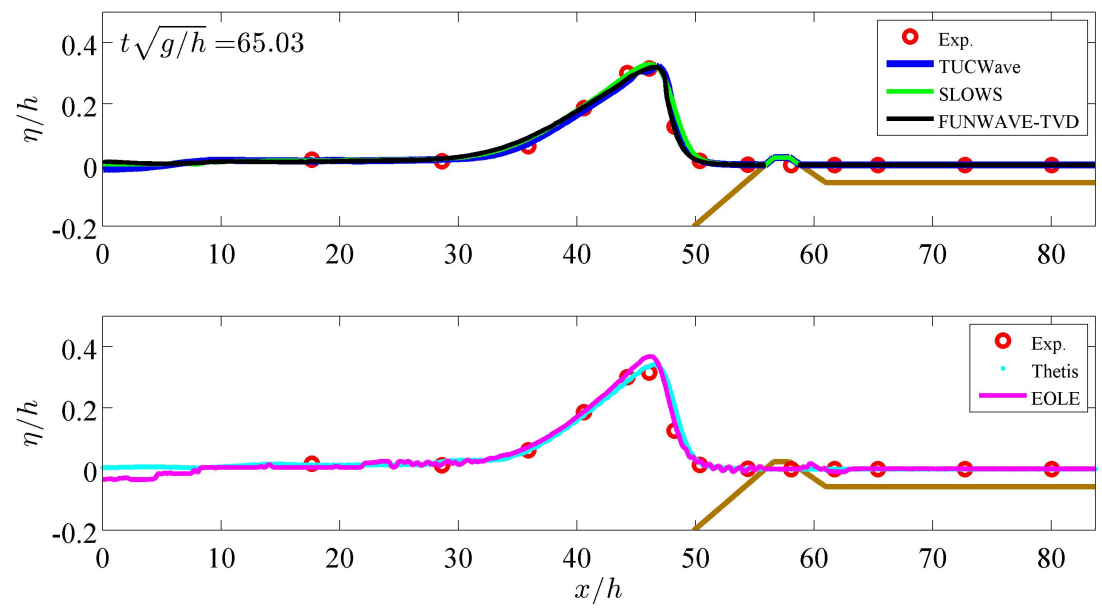

Figure 2(b) - Large scale flow behaviour. Water level at $\mathrm{t}^{*}=65.03$
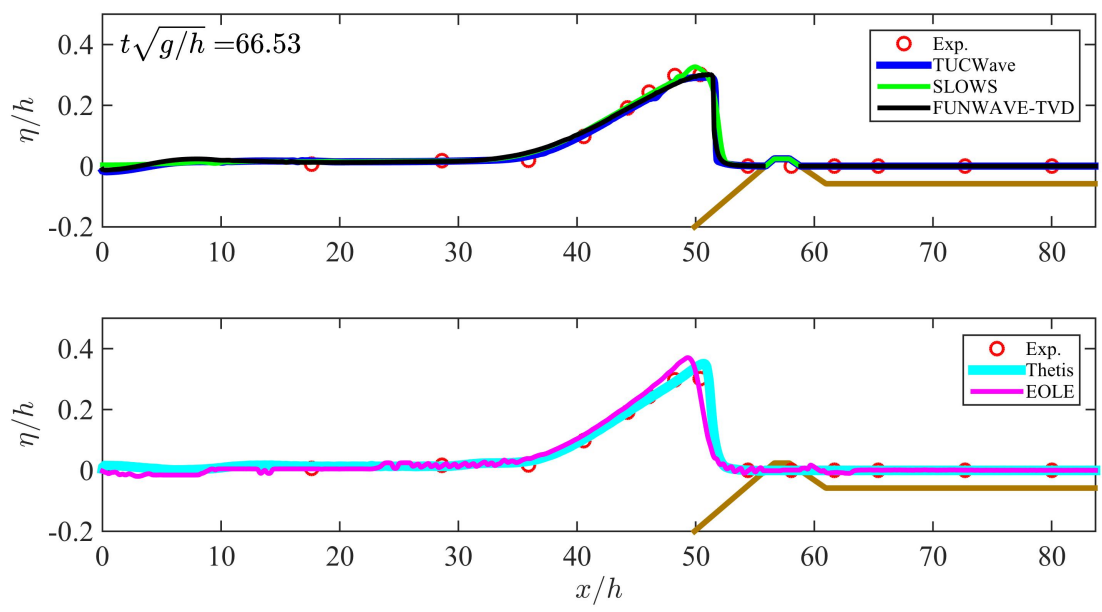

Figure 2(c) - Large scale flow behaviour. Water level at $t^{*}=66.53$

Figures 2(a), 2(b), and 2(c) show the propagation and shoaling of the solitary wave. The first figure shows the wave roughly in correspondence of the toe of the slope. At this time the wave is still quite symmetric. The loss of symmetry due to shoaling is clearly visible in figure 2(b), with incipient breaking conditions obtained in FUNWAVE-TVD already at $t^{*}=66.53$ in figure 2(c). For this set of snapshots all the models yield very close results. Minor differences can be seen in the sharper and slightly lower profile of FUNWAVE-TVD, especially in the last picture. This is due to the breaking treatment which has been activated, and which turns the wave into a moving bore. In figure 2(c) (and partially in figure 2(b)) we can also see that EOLE provides a peakier wave with regard to all the other models. This may be related to the post-processing of the Navier-Stokes results, as we will discuss later. All the codes agree satisfactorily with the data.

In the next set of figure (2(d) to 2(f)) the wave has broken and overtops the reef. It is known from the experiments that the wave develops into a plunging breaker. All the Boussinesq codes replaced this effect by a moving breaking front treated with the shallow water equations. Instead, the front observed in the figures in the second set is a result of the postprocessing of the Navier-Stokes codes. Despite of this, the models provide essentially similar results, especially at $t^{*}=69.13$ (figure $2(d)$ ) and $t^{*}=70.68$ (figure 2(e)). Visible differences are 
present instead in the last figure referring to time $t^{*}=72.48$ (figure 2(f)) at which the splashing of the breaker has occurred. The difference observed in the figure is a result of the different treatment of wave breaking. The depth-averaged models clearly provide sharp moving bores, with minor visible differences in the three results. This behaviour is due to the common wave breaking mechanism, which deactivates the dispersion at the wave front, when breaking is detected, and allows to conserve the water's total mass and momentum, while mimicking total energy dissipation via the dissipation in the shallow water shock. The position, and magnitude of the fronts is close to the experiments, validating the breaking detection and dissipation closure. The Navier-Stokes codes, which can resolve the finer scales of the flow, also identify moving fronts, which are more oscillatory when compared to the Boussinesq codes. These instabilities may be possibly artificially accentuated with the post-processing technique implemented to treat the complex flow pattern obtained in the splash-up phase, which we will discuss in the next section. What can be seen from figure 2(e) in this set of figures is that the post-processed data from EOLE accurately represent the plunging position of the wave and the resulting splash-up and bore formation. For the Thetis model, the amplitude of the breakers is well modelled, but the splash-up seems to occur earlier than with the other models. Despite of these differences, the overall agreement with the data is quite satisfactory for all the models.
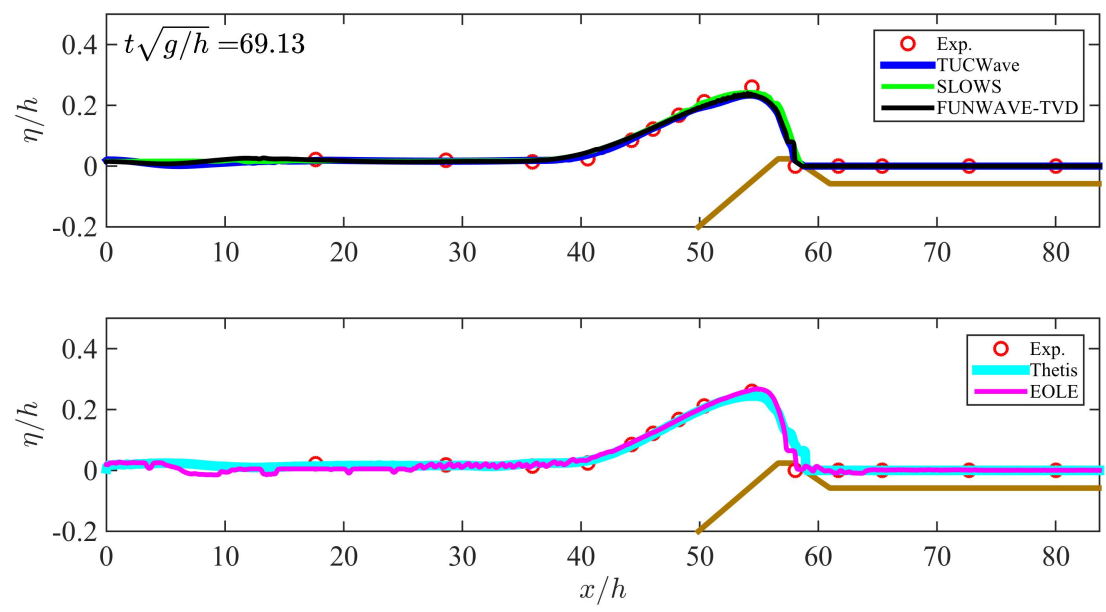

Figure 2(d) - Large scale flow behaviour. Water level at $\mathrm{t}^{*}=69.13$ 

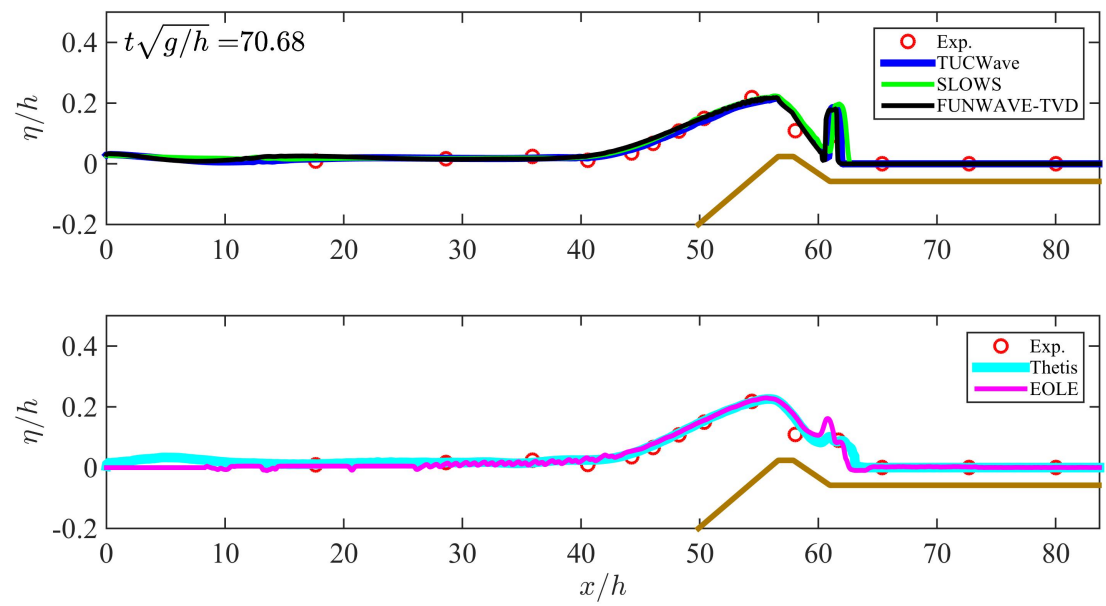

Figure 2(e) - Large scale flow behaviour. Water level at $\mathrm{t}^{*}=70.68$
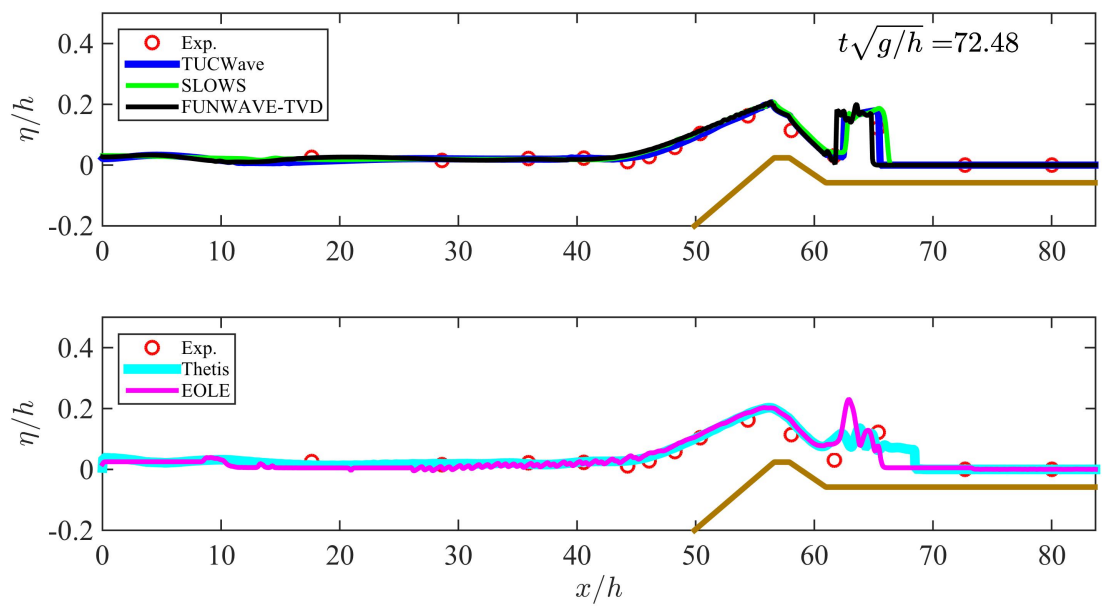

Figure 2(f) - Large scale flow behaviour. Water level at $\mathrm{t}^{*}=72.48$

The last set of pictures shows the evolution of the supercritical flow and of the bores developed onto the stagnant water. Laboratory observations indicate generation of a hydraulic jump with overturning of the free surface off the back reef and a turbulent bore propagating downstream (Roeber et al. 2012). The deforming bore propagates downstream showing a reduction in amplitude and is reflected by the wall at $\mathrm{t}^{*} \sim 99$. The reflected bore travels downstream and overtops the reef. At this point and as the water rushes down the fore reef, the flow transitions from flux to dispersion-dominated, and a hydraulic jump generated after the second overtopping transforms into an offshore propagating undular bore, ultimately giving a train of dispersive waves over the increasing water depth upstream. The development of this process, which we can see already starting in figure 2(f), can be observed in the results of figures 2(g) to 2(i). In the first two figures, we can see that the three Boussinesq codes provide again sharp moving bores, showing that the breaking model is still on. We can observe a visible phase lag with regard to experimental data for FUNWAVE, and a phase advance for SLOWS, and a more reduced one for TUCWave. Similarly to the previous 
pictures, the turbulent propagating bore is described in a more oscillatory way. This is certainly due to the post processing of the $2 \mathrm{D}$ flow. Thetis shows a considerable phase advance with regard to all the other models, while EOLE provide a front position in better agreement with the data. Amplitudes are however well described by the two models. Despite of its previous phase advance, Thetis provides an accurate description of the front position and amplitude after the reflection. EOLE gives a slighlty faster reflective wave, very comparable to TUCWave and SLOWS. Finally, after the second overtopping takes places, we can clearly in figure 2(i) see the undulating bore forming in the results of SLOWS and TUCWave, and in those of EOLE. Both FUNWAVE and Thetis fail in providing a description of this feature, which may be related to the breaking detection technique used in FUNWAVE, and in a lack of resolution in the computations performed with Thetis.
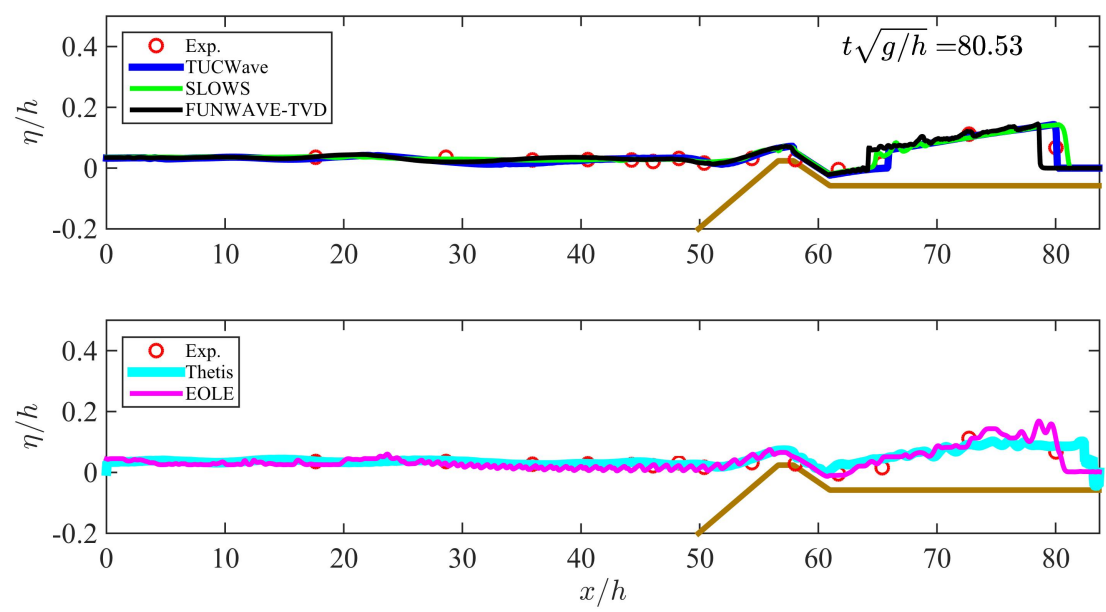

Figure $2(\mathrm{~g})-$ Large scale flow behaviour. Water level at $\mathrm{t}^{*}=80.53$
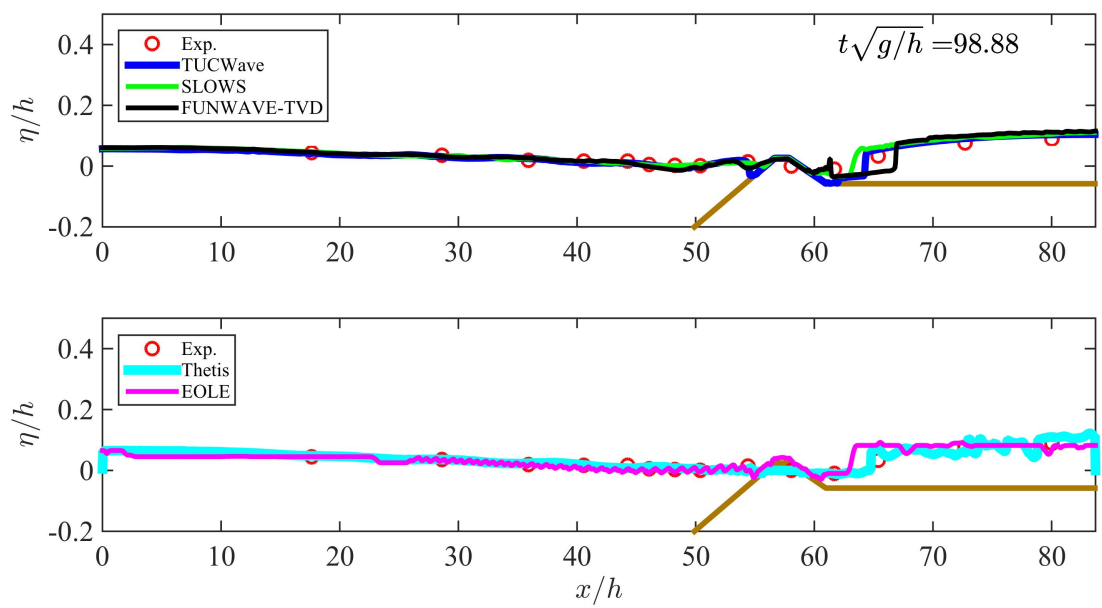

Figure 2(h) - Large scale flow behaviour. Water level at $\mathrm{t}^{*}=98.88$ 

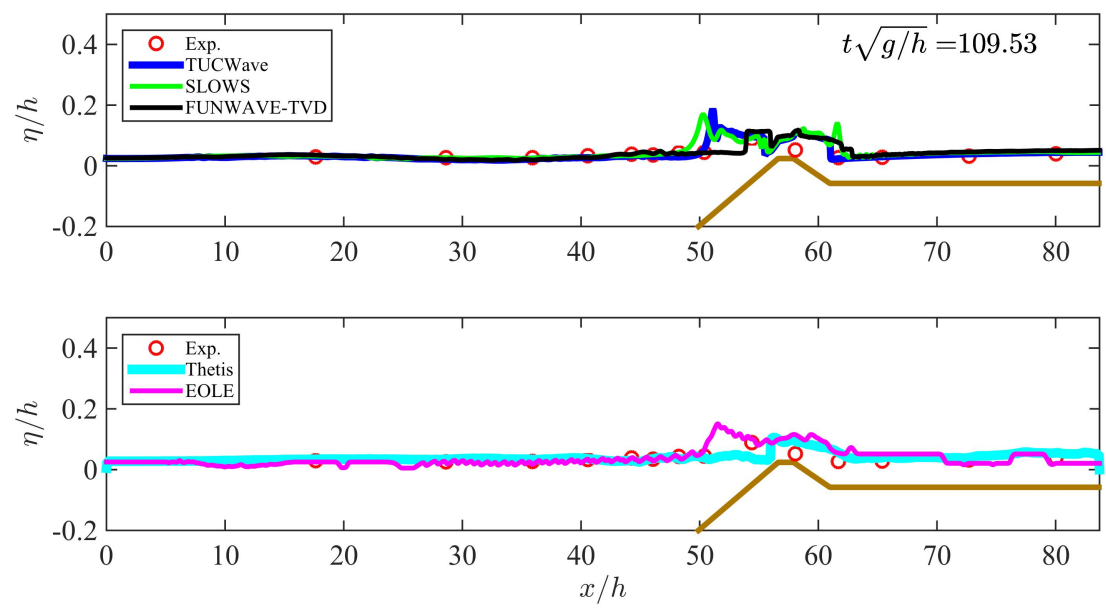

Figure 2(i) - Large scale flow behaviour. Water level at $\mathrm{t}^{*}=109.53$

We consider now the numerical and experimental time series of the water height in the wave gauges. We will analyse 5 of the 14 gauges. The computed and experimental series corresponding to these probes are reported in figures 3(a) to 3(e). These five gauges allow to somehow single out some of the features of the computed flows, namely:

- WG2 is representative of the initial propagation and final dispersive phases;

- WG5 is representative of the shoaling and final dispersion dominated phase;

- WG9 is representative of the breaking phase and of the formation of the hydraulic jump on the fore reef side;

- WG10 is representative of the overtopping phases;

- WG12 is representative of the propagation of the initial and reflected bores.

Overall, all the models are able to catch-up the flow behaviour, but a closer look reveals some interesting differences. In the propagation phase, we can see from the WG2 data that, while all Boussinesq-type models provide an accurate approximation of the solitary wave movement toward the reef, both the Navier-Stokes solvers overestimate the height of the wave. Moreover, with the resolutions used in the computations both Navier-Stokes simulations seem to miss the higher frequency modes present in the Boussinesq results. These, however, while giving a reasonable reproduction of the amplitude of these modes, all suffer from phase errors, which may be due to the limitations in dispersion accuracy of the models and on the different wave breaking treatments used. The time series in WG5 lead to similar conclusions. In particular, both Navier-Stokes models overestimate the shoaling height, which is well reproduced by all Boussinesq simulations. More importantly, the Navier-Stokes results fail to reproduce the undulating bore originated by the reflected wave overtopping the reef. The EOLE results only capture the first peak, and miss the remaining secondary oscillations. Thetis fails completely to catch this feature. Among the Boussinesq models, one can see that TUCWave overestimates some of the oscillations, as expected with the type of model used in this code. In the breaking phase we can see from WG9 (figure 3(c)) data that all models are able to catch the vertical front of the wave. The BT models underestimate the wave height with FUNWAVE-TVD giving a slightly lower profile compared to the other two BT models due to the different breaking treatment used. Again 
both the Navier-Stokes solvers overestimate the height of the wave. Further in time and for the BT models we can see the formation of the undular bore with a visible phase lag with regard to experimental data for FUNWAVE-TVD and a phase advance for SLOWS and TUCWave. EOLE model reproduces the bore satisfactorily but Thetis fails to reproduce the formation of the undular bore maybe due to overestimation of wave energy dissipation over the breakwater.
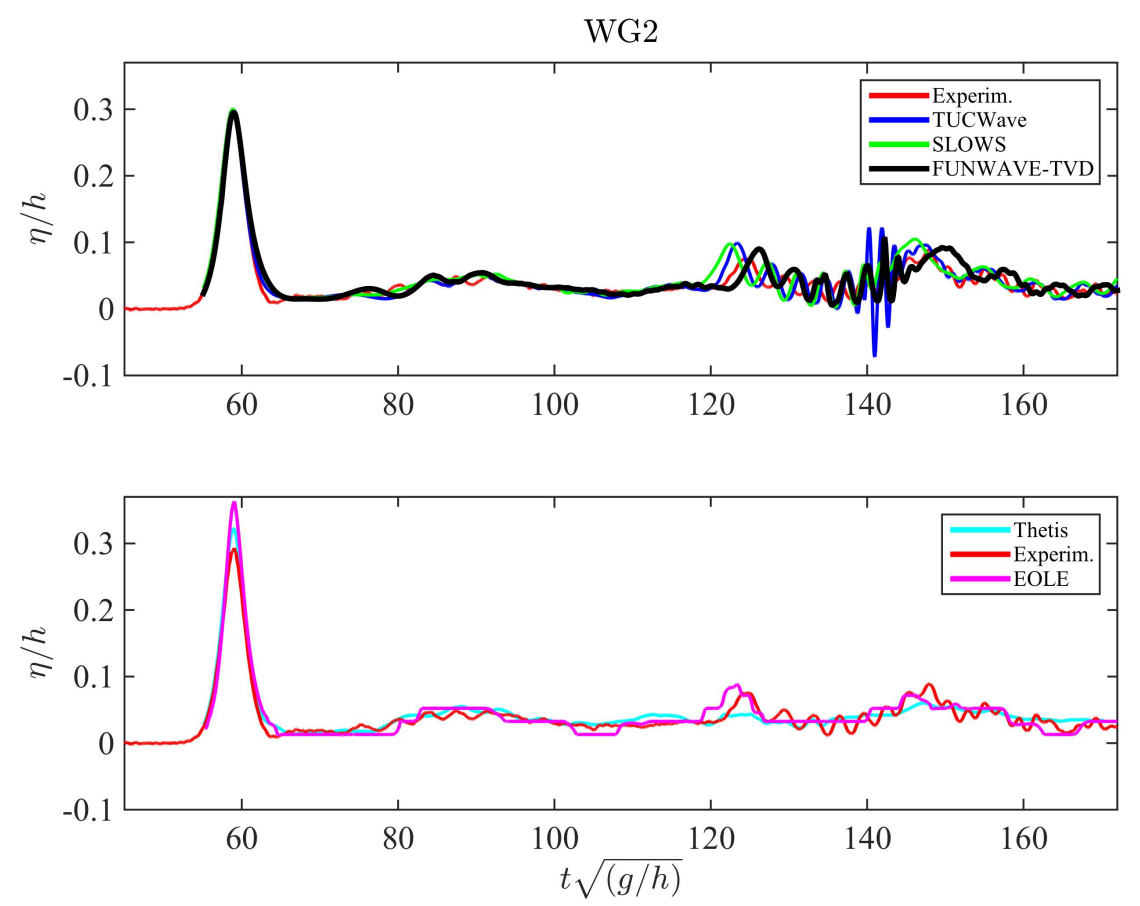

Figure 3(a) - Large scale flow behaviour. Time series in WG2 (propagation) 
WG5
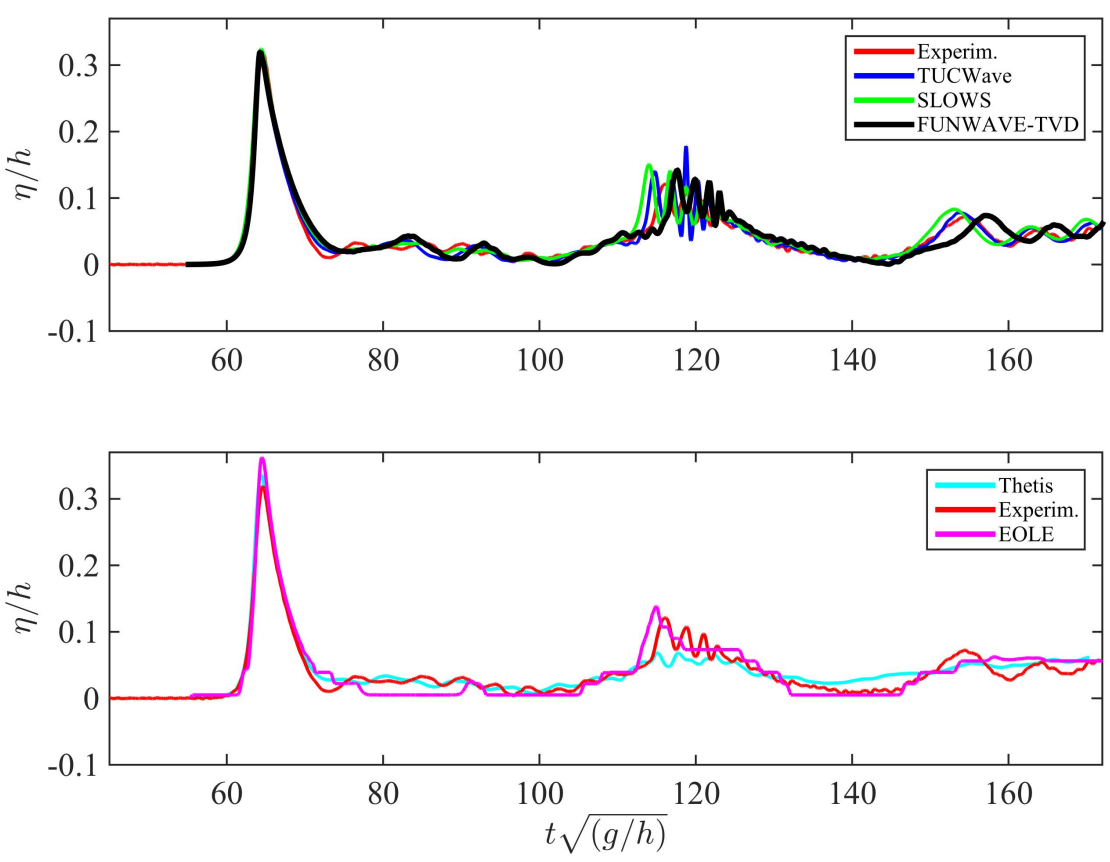

Figure 3(b) - Large scale flow behaviour. Time series in WG5 (shoaling)
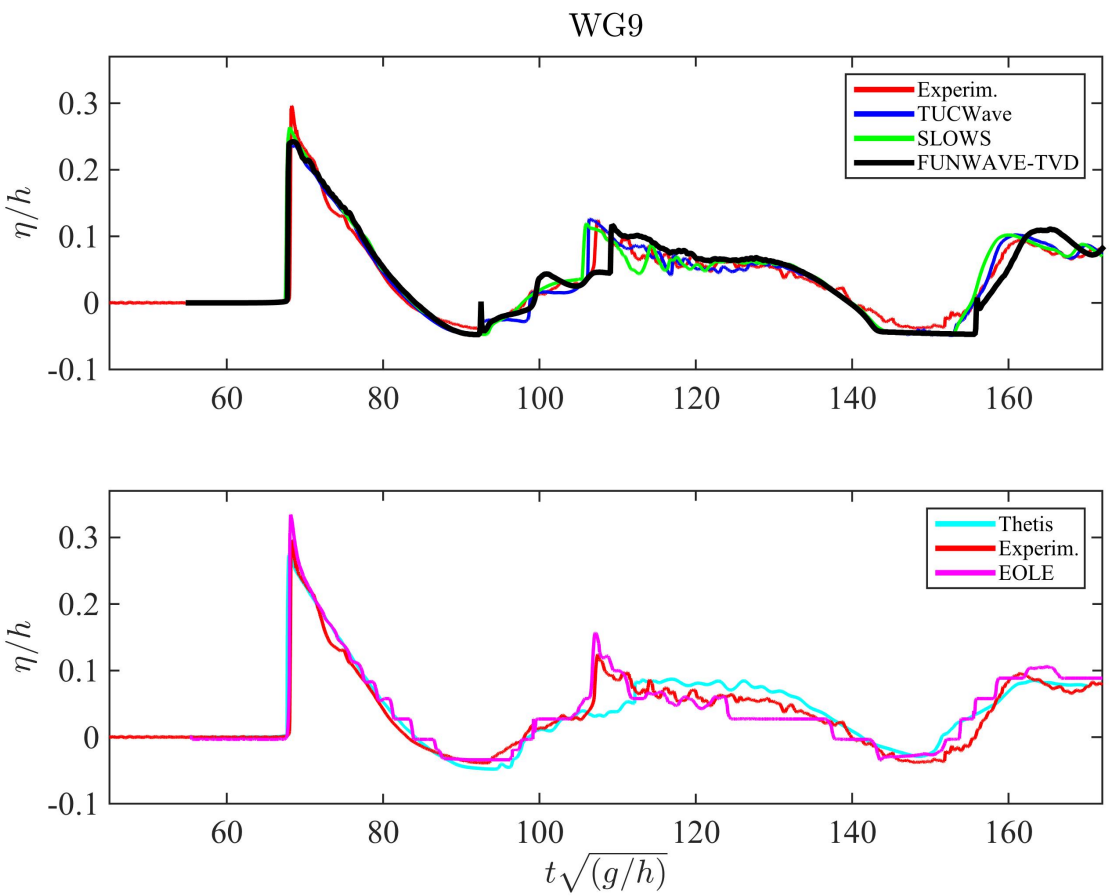

Figure 3(c) - Large scale flow behaviour. Time series in WG9 (breaking) 
The time series relative to WG10 are reported on figures 3(d). WG10 is placed on top of the reef and the time series show the overtopping of the first wave, whose amplitude is overestimated by all models, and then the drought of the area, which cannot be reproduced by FUNWAVE-TVD, and EOLE for which the remaining thin water layer is probably due to a lack of grid refinement in this zone. The second overtopping that occurs from the reflected (to the right wall) bore is also overestimated for all codes.
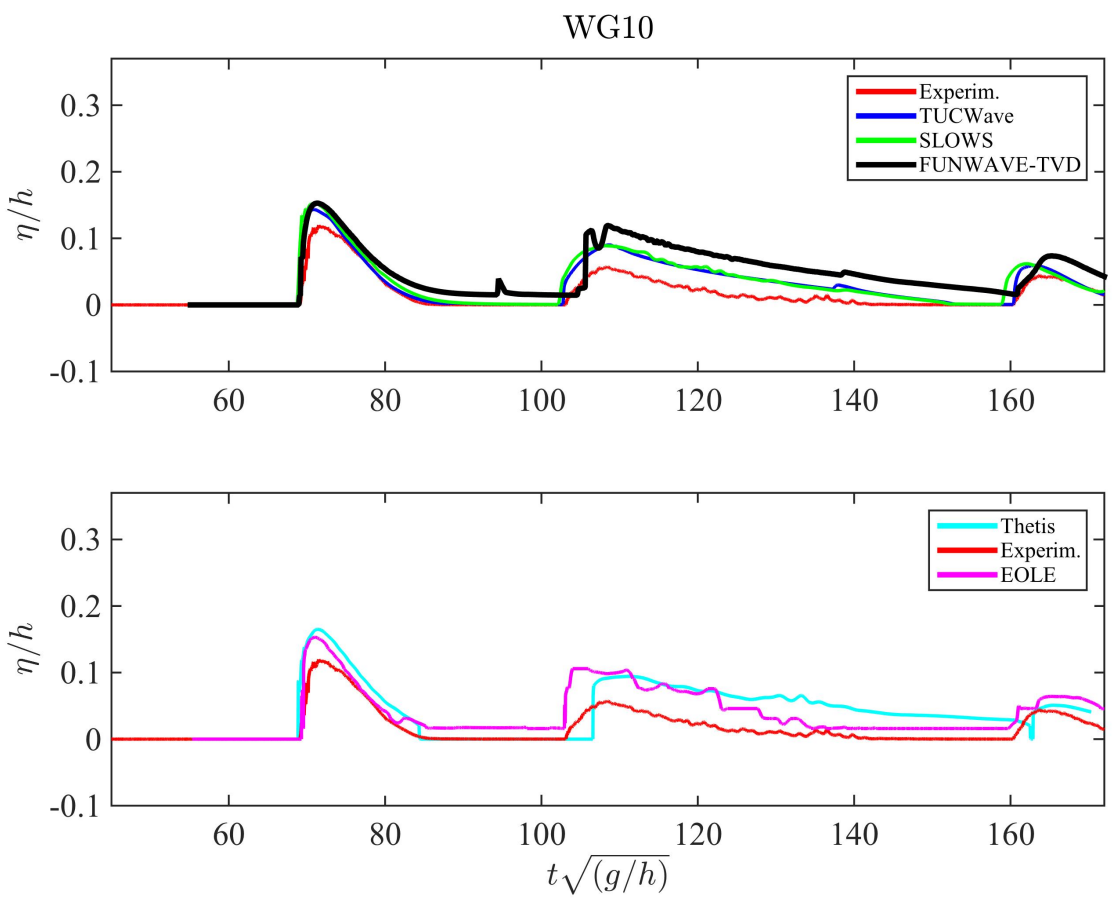

Figure 3(d) - Large scale flow behaviour. Time series in WG10 (overtopping)

Finally, figure 3(e) reports the time series relative to WG12. For the BT models we can see that all models overestimate the initial wave, but the reflected bores are well-reproduced with regard to experimental data, expect a small phase lag on the second wave for the FUNWAVE-TVD model. The numerical results produced by SLOWS are more oscillatory after each propagating bore, which are simulated as moving shocks, due to the nature of the wave breaking mechanism used. In the second set of figures, we can see that even though the Navier-Stokes solvers are able to simulate the amplitude of the first wave better with regard to BT models, their numerical results deviate from the experimental data after the second reflected wave.

As a next step, we compute the Root Mean Square Deviation (RMSD) in order to measure the differences between the numerical results and the experimental data for each wave gauge. Of course this study shows only a qualitative appreciation of the models since we do not have any error information for the experimental data. Table 1 and figure 4 present the RMSD for each wave gauge and for each model. 

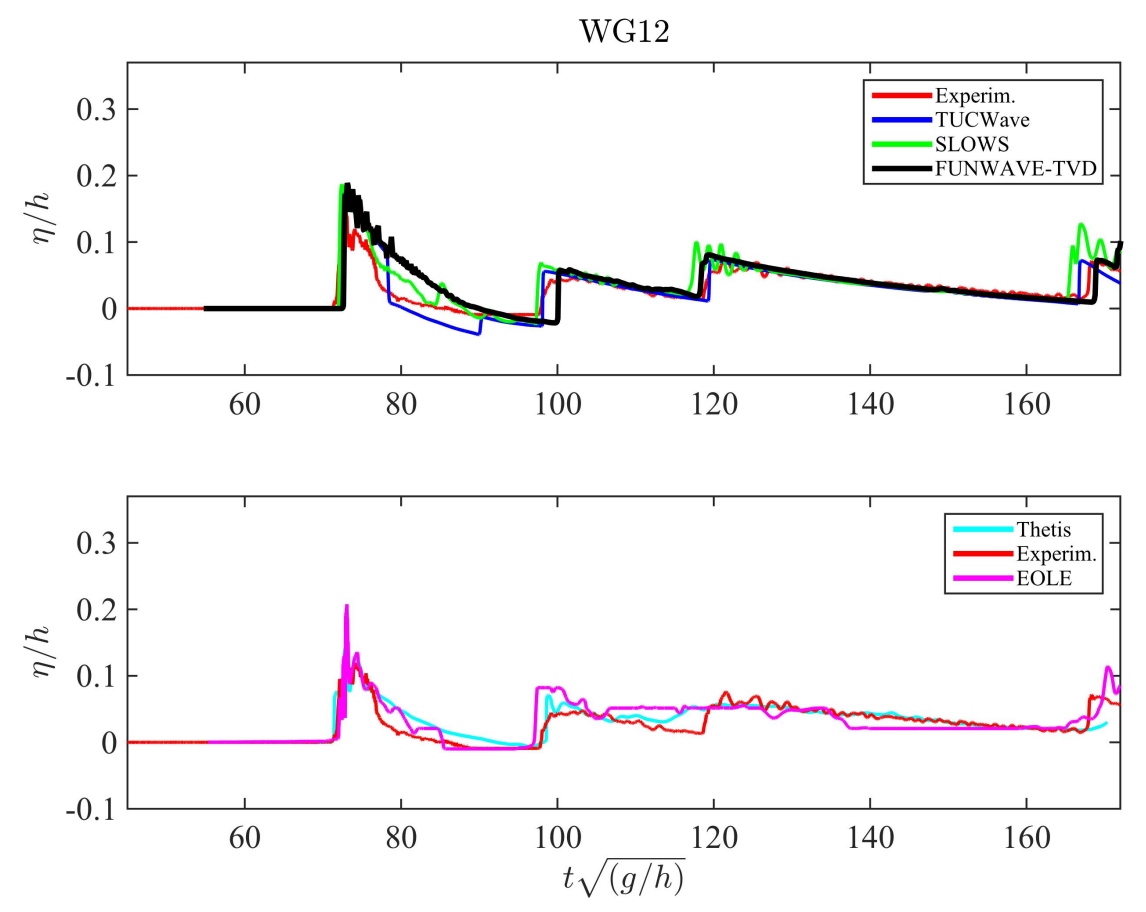

Figure 3(e) - Large scale flow behaviour. Time series in WG12 (bore propagation)

Overall, all the models give the same order of RMSD, especially for the wave gauges placed before the reef, indicating that all models correctly handle propagation and shoaling of the solitary wave. A closer look at the gauges before the reef reveals that the BT models present a bigger RMSD in the first three gauges with regard to Navier-Stokes solvers for which Thetis code presents the lower RMSD values. The RMSD of the next four wave gauges (WG5 WG8) for the Navier-Stokes computations grows and is bigger with regard to BT models. This can be attributed to the fact that both Navier-Stokes computations miss the higher frequency modes, which are present in the Boussinesq results. In WG9, Thetis presents the bigger RMSD since it fails to reproduce accurately the undular bore originated by the reflected wave overtopping the reef. The RMSD of the several models vary the most for WG10. This wave gauge indicates the correct wet/dry treatment of the models. FUNWAVETVD has the maximum value and TUCWave has the minimum. The next three gauges (WG11-WG13) are representative of the propagation of the initial and reflected bores and we observe more or less the same behavior. Boussinesq models provide almost the same RMSD value, while the Navier-Stokes solvers have a visible difference between them, with Thetis giving the minimum RMSD. This may be related to the post-processing of the Navier-Stokes results, as we will discuss in the next section. The RMSD value for the last wave gauge WG14 indicates in part the correct wall boundary treatment and in part the correct dissipation rate. In this case, Thetis presents the maximum value.

To conclude, we present the CPU time needed for each model in Table 3. The CPU time has been measured for a simulation period of 100s. However note that the computations have been run on different machines, using different programming languages, compilers etc. So these figures are to be taken as a very qualitative indication of the relative cost of the models. 


\begin{tabular}{|c|c|c|c|c|c|}
\hline & EOLE & $\begin{array}{l}\text { FUNWAVE } \\
\text { TVD }\end{array}$ & SLOWS & THETIS & TUCWave \\
\hline WG2 & 0.0124 & 0.0131 & 0.0150 & 0.0108 & 0.0157 \\
\hline WG3 & 0.0114 & 0.0130 & 0.0144 & 0.0122 & 0.0145 \\
\hline WG4 & 0.0130 & 0.0126 & 0.0131 & 0.0121 & 0.0132 \\
\hline WG5 & 0.0144 & 0.0139 & 0.0127 & 0.0140 & 0.0128 \\
\hline WG6 & 0.0145 & 0.0138 & 0.0128 & 0.0143 & 0.0125 \\
\hline WG7 & 0.0162 & 0.0132 & 0.0133 & 0.0158 & 0.0120 \\
\hline WG8 & 0.0177 & 0.0149 & 0.0145 & 0.0180 & 0.0147 \\
\hline WG9 & 0.0191 & 0.0185 & 0.0190 & 0.0235 & 0.0179 \\
\hline WG10 & 0.0291 & 0.0374 & 0.0229 & 0.0338 & 0.0188 \\
\hline WG11 & 0.0253 & 0.0210 & 0.0223 & 0.0186 & 0.0227 \\
\hline WG12 & 0.0208 & 0.0190 & 0.0198 & 0.0143 & 0.0163 \\
\hline WG13 & 0.0204 & 0.0159 & 0.0167 & 0.0144 & 0.0114 \\
\hline WG14 & 0.0226 & 0.0206 & 0.0148 & 0.0269 & 0.0146 \\
\hline
\end{tabular}

Table 2 - RMSD values for each model

\begin{tabular}{|c|c|c|c|c|c|}
\hline & EOLE & $\begin{array}{l}\text { FUNWAVE } \\
\text { TVD }\end{array}$ & SLOWS & THETIS & TUCWave \\
\hline CPU time & $1 d$ and $6 h$ & $250 \mathrm{sec}$ & $71.37 \mathrm{sec}$ & $1 \mathrm{~d}$ and $4 \mathrm{~h}$ & $41.33 \mathrm{sec}$ \\
\hline Machine & $\begin{array}{l}\text { Linux Redhat } \\
5,2 \text { processors } \\
\text { (Intel } \AA \text { Xeon } \\
\text { ( X X7460) } 6 \\
\text { cores (2.66 } \\
\text { GHz), hyper } \\
\text { threading (so } \\
24 \text { threads) }\end{array}$ & $\begin{array}{l}1 \text { single } \\
\text { processor } \\
\text { (AMD Abu } \\
\text { Dhabi } 2.6 \mathrm{GHz} \text { ) }\end{array}$ & $\begin{array}{l}\text { IMac } 3.5 \mathrm{Gz} \text {, } \\
\text { Intel }{ }^{\circledR} \text { Core i } 7\end{array}$ & $\begin{array}{l}2 \times 8 \\
\text { processors, } \\
\text { Nodes - } \\
\text { C6100 (x264) } \\
2 \text { processors } \\
6 \text { cores }(12 \\
\text { cores/node) } \\
3,06 \mathrm{GHz}\end{array}$ & $\begin{array}{l}\text { IMac } 3.5 \mathrm{Gz}, \\
\text { Intel }{ }^{\circledR} \text { Core } 17\end{array}$ \\
\hline
\end{tabular}




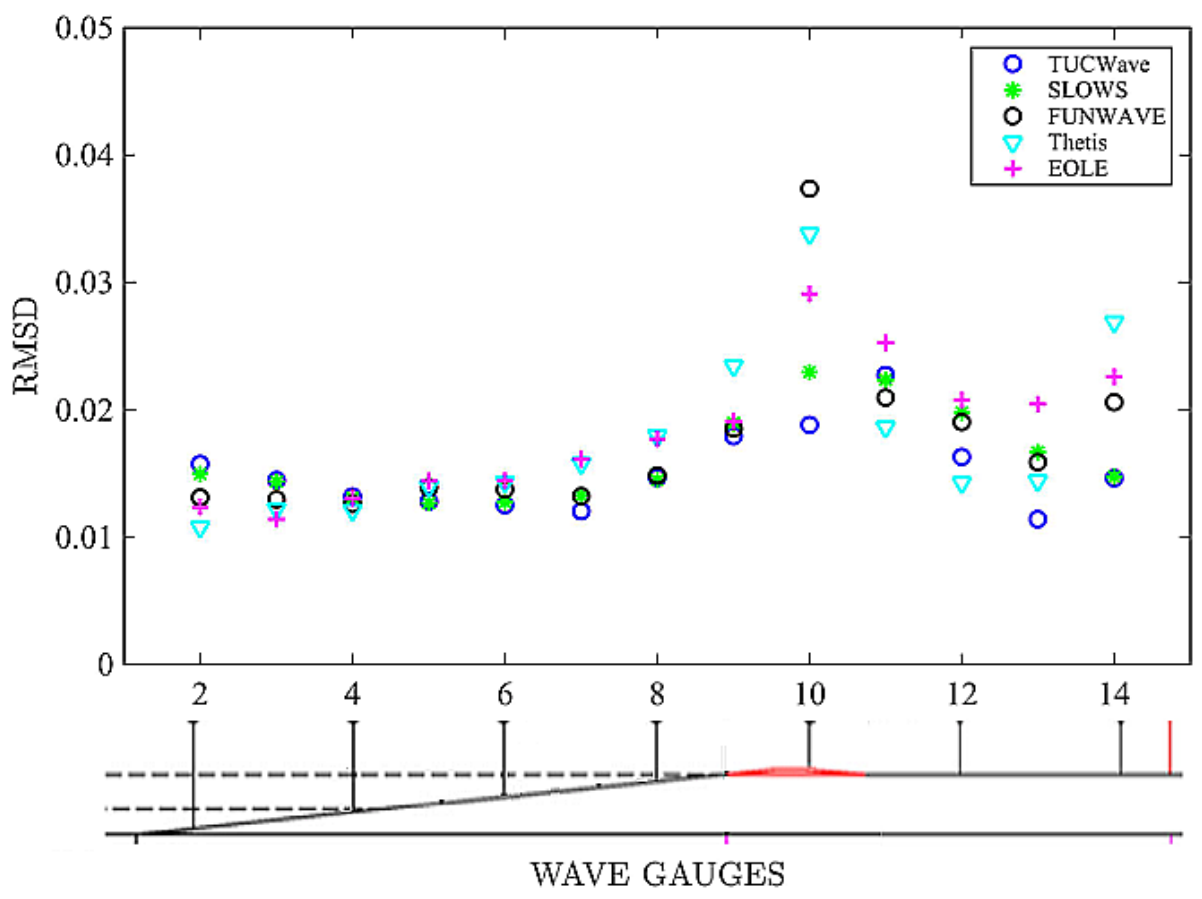

Figure 4 -RMSD in the wave gauges for each model (with a rough sketch of the gauge position)

\section{Finer scale features and post-processing}

A comparison of the measured and computed wave profiles is first presented in figure . For Navier-Stokes VOF codes, it is necessary to introduce a specific post-treatment algorithm allowing to localize the free surface elevation at each gauge. For exemple in EOLE, for a given position of the gauge the procedure sweeps vertically the VOF field (i.e., the water volume fraction) in all the cells until it detects a partial $\mathrm{VOF}$ value $(0<\mathrm{VOF}<1)$ meaning the cell containing the interface (knowing that $\mathrm{VOF}=1$ and $\mathrm{VOF}=0$ mean respectively purely liquid and purely air). From the vertical coordinates of the detected cell and its own VOF value a position of the free surface is extracted. The weakness of this algorithm is that the accuracy is strongly meshing size dependence. So the issue is not an exact position of the interface but rather a mean position with an error rounded to the mesh cell size. Other problems may be encountered as well when there are possibly several partial volume fraction values along the same vertical, for instance break-up of droplets from the free surface or air bubble entrainment during splash-up phenomenon (see for example figure 5).

For THETIS, same kind of problems are encountered. Indeed the flow and the water/air interface is very complex in this benchmark due to the strong mixing generated by wave breaking (figure 6). For this reason, the processing of the water/air interface needs to be carefully analysed in Navier-Stokes equations because these models capture at least a certain part of this complexity. This is illustrated in figure 6 which presents the bore propagation over the reservoirs computed by THETIS. White contours represent different fraction of water in cells $(10 \%, 50 \%$ and $90 \%)$. As shown in this figure, the bore is highly mixed. This has two repercussions: first, the density is equally affected by this mixing and this may play a role in the model behaviour, second the identification of the surface elevation is complicated as no 
actual interface can be defined as in Boussinesq models for instance in which this elevation is one of the model parameter. The results presented in this benchmark were computed with a free surface corresponding to $F=0,5$ (see section 3.3). The following figure 7 shows that this is only a limited view of the available information. Note that this remark also holds for the experimental measurements.

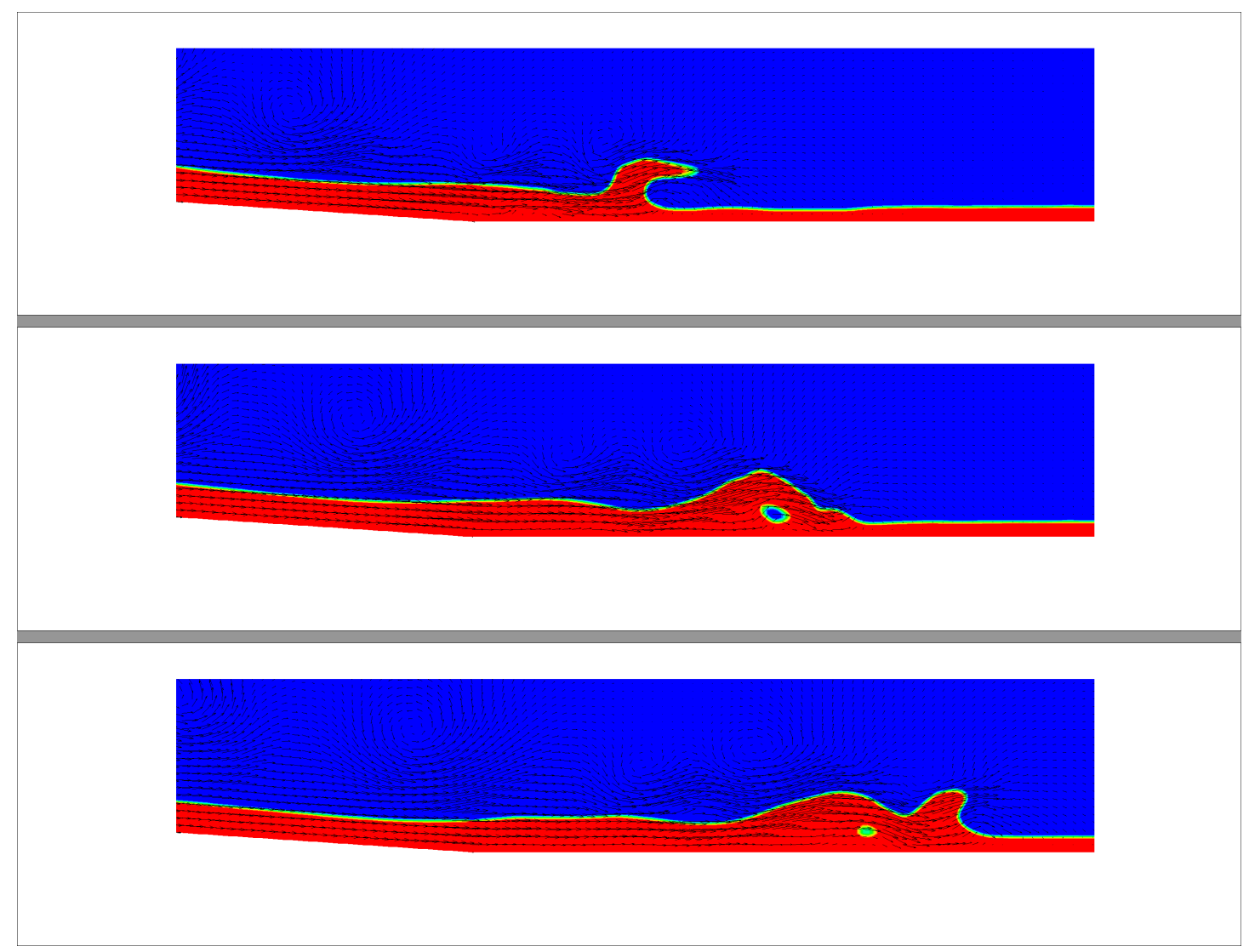

Figure 5: splash-up and air bubble entrainment processes computed by the EOLE model
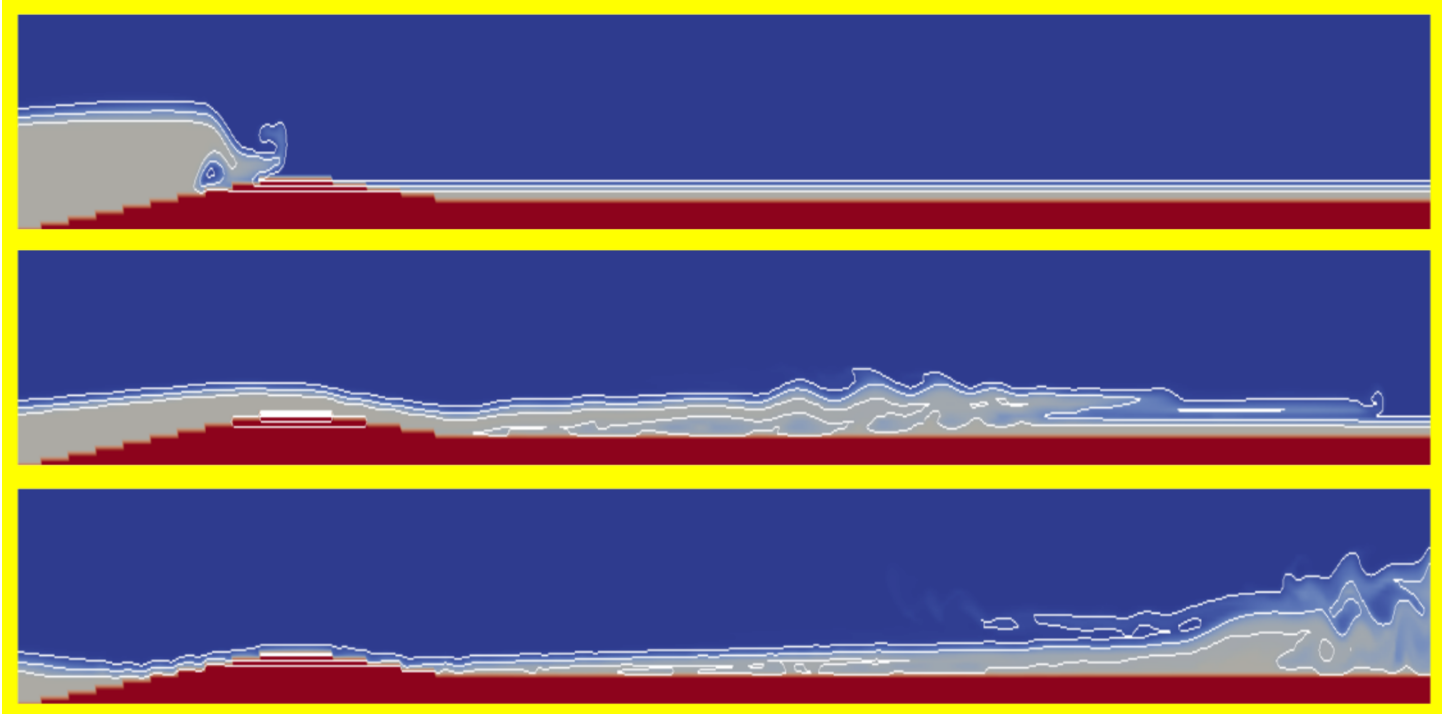

Figure 6: plunging breaking and splash-ups computed by the Thetis model 


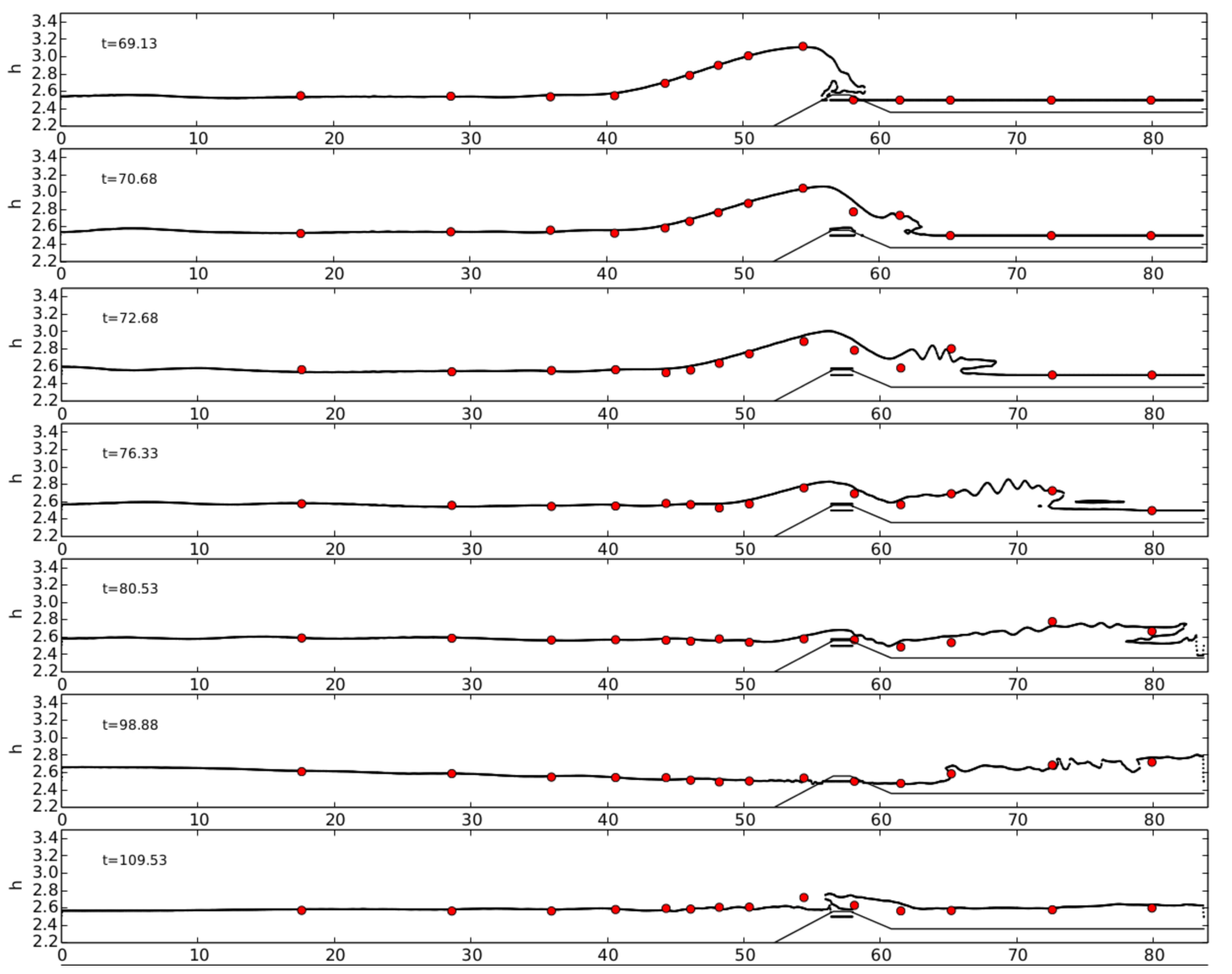

Figure 7: Snaphots of experimental free surface and water/air interface (i.e., F=0.5 computed by Thetis.

\section{Summary and conclusions}

- Thetis code has the best RMSD for the first three wave gauges where the propagation of the solitary wave, going on-shore, and the propagation of the undular bore, going off-shore, are depicted. Looking closely at the time series of those wave gauges we can see the perfect match of the computational and the experimental data for the solitary wave that travels on-shore while there is a deviation especially on the front of the undular bore, under-predicting the wave's amplitude. During the propagation of the bore on the lake (after the reef) we observe a mismatch of the numerical and experimental data, probably accented by some inaccuracies regarding the surface elevation post-processing treatment and a larger wave energy dissipation than measured.

- EOLE code presents a quite similar behaviour as the Thetis code, especially at the beginning of the process. But if the height of the wave is slightly more overestimated in the first step of the propagation, a better approximation of the wave's splash-up and associated processes is then observed. Note that, as previously said, the 
experiment doesn't allow to qualify accurately the corresponding oscillations of the free surface which occur at the wave's splash-up. On the whole, this code provides with a satisfying description of the process, especially of the early phase, and a good prediction of the dispersion dominated undular bore, but with higher frequency oscillations missing in the results probably due to a lack of resolution.

- TUCWave code run with parameter values for CFL 0.2 and for the breaking criterion $\gamma=0.6$. The code accurately predicts the propagation of the wave across the channel. During shoaling it slightly over predicts the wave's amplitude, which is expected since the code solves the weakly non-linear weakly dispersive equations of Nwogu (1993). The numerical model accurately predict both time and place of the wave breaking and mimics the breaker as a collapsing bore. The wet/dry front is also accurately simulated since TUCWave is the only code manages to give an error less that $0.02 \%$ in wave gauge 10 (where dry reef is placed). The limitation in describing the amplitude of the undular bore deformed after the overtopping of the reflected bore can be attributed to the nature of the equations solved along with the wave breaking technique used.

- SLOWS solves the GN equations with a CFL parameter of 0.2 and $\gamma=0.6$ for the breaking criterion. The results are similar to the ones provided by the two others Boussinesq-type models. Like before the breaker is simulated as a collapsing bore that slightly underestimates the wave height but conserves the total mass. We can observe a very good much of the experimental and the numerical data for the undular bore and the dispersive waves that produced as it travels off-shore.

- FUNWAVE -TVD gives almost the same results as the two previous Boussinesqtype models. A time lag between the numerical results and the experimental data is observed during the propagation of the bore on the lake and afterwards.

In conclusion, the five codes can be classified in two main categories. Those based on the Naver-Stokes (NS) model (Thesis and EOLE) and the depth-averaged Boussinesq-type (BT) models (TUCwave, SLOWS, FUNWAVE-TVD). Surprisingly both categories provide very similar and satisfactory results on the whole (according to experiment), in particular a good agreement is observed between EOLE's, SLOW's and TUCWave's results. The computational times of the BT models are significantly less compared to the ones of the NS models. Hence, from the practical point of view, there is no a huge advantage from the NS models compared to the BT models. But we have to keep in mind that the amount of information obtained from NS models is significantly more than the one obtained from the BT models. Among the BT models the results are comparable but there are some differences concerning mainly the phase lag observed. This difference is expected and can be attributed to the fact that the BT models with the hybrid wave breaking closure are very sensitive to the trigger mechanism, which is different in each code. Additionally the trigger mechanism depends on the hydrodynamic quantities provided by the models, which are not the same due to the different nature of the models used. Further more a very crucial point is that we don't have any error measurement on the experimental results (very few experiments exists in the literature) and we can't quantify the behaviour of the codes.

This demanding test case has allowed gaining insight in the capabilities and limitations of five numerical codes aimed at modelling tsunami propagation over long distances. In this test case multiple wave effects have been considered, as propagation, shoaling due to bottom variations, breaking, reflections and wet-dry front interactions. 


\section{Acknowledgements}

This comparative study has been performed within the Work Package 1 (Benchmarking of numerical methods) of the TANDEM project (Tsunamis in the Atlantic and the English ChaNnel: Definition of the Effects through numerical Modelling) (2014-2018), with partial funding from the PIA-ANR program (Programme Investissements d'Avenir, Agence Nationale de la Recherche) from the French Government under grant 2013-RSNR-01.

\section{References}

Abadie, S., Caltagirone, J.-P., Watremez, P. (1998) Splash-up generation in a plunging breaker. Comptes Rendus de l'Académie des Sciences - sériés IIB, 326:556-559.

Abadie, S., Morichon, D., Grilli, S., Glockner, S. (2010) Numerical simulation of waves generated by landslides using a multiple-fluid Navier-Stokes model. Coastal Engineering 57: 779-794.

Biausser, B., Guignard, S., Marcer, R., Fraunié, P. (2004) 3-D two phase flows numerical simulations by SL-VOF method, Int. J. Numer. Meth. Fluids; 45:581-604.

Biausser, B., Marcer, R., Grilli, S., Fraunié, P. (2004) Numerical Analysis of the Internal Kinematics and Dynamics of 3-D Breaking Waves on Slopes, International Journal of Offshore and Polar Engineering, Vol. 14, No. 4, December 2004 (ISSN 1053-5381).

Brackbill, J. U., Kothe, D. B., \& Zemach, C. (1992). A continuum method for modeling surface tension. Journal of computational physics, 100(2), 335-354.

Desombre, J, Morichon, J.D., Mory, M. (2013) RANS v2-f simulation of a swash event: Detailed flow structure. Coastal Engineering, 71:1-12.

Filippini, A.G., Kazolea, M., Ricchiuto, M. (2016) A flexible genuinely nonlinear approach for nonlinear wave propagation, breaking and run-up. J. Comp. Phys., 310:381-417.

Fortin, M., Glowinski, R. (1982) Méthodes de lagrangien augmenté: applications à la résolution numérique de problèmes aux limites. Dunod.

Grilli, S.T., Harris, J.C., Tajalli Bakhsh, T.S., Masterlark, T.L., Kyriakopoulos, C., Kirby, J.T. and Shi, F. (2013) Numerical Simulation of the 2011 Tohoku Tsunami Based on a New Transient FEM Co-seismic Source: Comparison to Far- and Near-Field Observations, Pure and Applied Geophysics, 170, 1333-1359.

Guignard, S., Marcer, R., Rey, V., Kharif, C., Fraunié, P. (2001) Solitary wave breaking on sloping beaches : 2D two phase flow numerical simulation by SL-VOF method, Eur. J. Mech. B, Fluids 20 (2001) 57-74.

Kazolea M., Delis A.I. (2013) A well balanced shock-capturing hybrid finite volume-finite difference numerical scheme for extended 1D Boussinesq models, Appl.Num. Math., 67:167186.

Kazolea M., Delis A.I., Nikolos I.K., Synolakis C.E. (2012) An unstructured finite volume numerical scheme for extended Boussinesq-type equations. Coastal Engineering, 69, 42-66. 
Kazolea M., Delis A.I., K., Synolakis C.E. (2014) Numerical treatment of wave breaking on unstructured finite volume approximations for extended Boussinesq-type equations. J.Comp.Phys., 271:281-305.

Kirby, J.T., Shi, F., Harris, J.C., Grilli, S.T. (2013) Dispersive tsunami waves in the ocean: Model equations and sensitivity to dispersion and Coriolis effects. Ocean Modelling, 62:3955.

Li, J. (1995) Piecewise Linear Interface Calculation. C.R.Acad.Sci, Paris, IIb, 320, 391-396.

Lubin, P., Vincent, S., Abadie, S., Caltagirone, J.-P. (2006) Three-dimensional Large Eddy Simulation of air entrainment under plunging breaking waves. Coastal Engineering 53, 631655.

Marcer, R., Journeau, C., Pons, K. (2016) Tsunami modelling. Validation of EOLE CFD code on academic test cases, XIVèmes Journées Nationales Génie Côtier - Génie Civil, Toulon, 29 juin au $1^{\text {er }}$ juillet 2016.

Mory, M., Abadie, S., Mauriet, S., Lubin, P. (2010) Run-up flow of a collapsing bore over a beach. European Journal of Mechanics - B/Fluids ,doi:10.1016/j.euromechflu.2010.11.005.

Nowgu, O. (1993) Alternative form of Boussinesq equations for nearshore wave propagation. Journal of Waterway, Port, Coastal and Ocean Engineering. ASCE, 119 (6), 618-638.

Patankar, S.(1980) Numerical heat transfer and fluid flow. CRC Press.

Pianet, G., Vincent, S., Leboi, J., Caltagirone, J. P., Anderhuber, M. (2010) Simulating compressible gas bubbles with a smooth volume tracking 1-fluid method. International Journal of Multiphase Flow, 36(4), 273-283.

Ricchiuto, M. (2015) An explicit residual based approach for shallow water flows. J. Comp. Phys, 280, 306-344.

Ricchiuto, M., Filippini, A.G. (2014) Upwind residual discretizations of enhanced Boussinwsq equations for wave propagation over complex bathymetries. J. Comp. Phys., 271:306-341.

Roeber, V., Cheung, K.F., Kobayasi, M.H. (2010) Shock-capturing Boussinesq-type model for nearshore wave processes. Coastal Engineering, 57:407-423.

Roeber, V., Cheung, K.F. (2012) Boussinesq-type model for energetic breaking waves in fringing reef environments. Coastal Engineering, 70:1-20.

Roeber, V., (2010) Boussinesq-type model for nearshore wave processes in fringing reef environment, $\mathrm{PhD}$ thesis, University of Hawaii.

Sagaut, P. (1998). Introduction à la simulation des grandes échelles pour les écoulements de fluide incompressible (Vol. 30). Springer Science \& Business Media.

Shi, F., Kirby, J.T., Harris, J.C., Geiman, J.D. and Grilli, S.T. (2012) A high-order adaptive time-stepping TVD solver for Boussinesq modeling of breaking waves and coastal inundation. Ocean Modelling, 43-44:36-51.

Tehranirad, B., Shi, F., Kirby, J.T., Harris, J. C. and Grilli, S.T. (2011) Tsunami benchmark results for fully nonlinear Boussinesq wave model FUNWAVE-TVD, Version 1.0. Technical report, No. CACR-11-02, Center for Applied Coastal Research, University of Delaware. 
Tonelli, M., Petti, M. (2013) Numerical simulation of wave overtopping at coastal dikes and low-crested structures by means of a shock-capturing Boussinesq model, Coastal Engineering, 79:75-88.

Wei, G., Kirby, J.T., Grilli, S.T., Subramanya, R. (1995) A fully nonlinear Boussinesq model for surface waves. I. Highly nonlinear, unsteady waves. J.Fluid Mechanics 294:71-92. 